\title{
The Involvement of Arl-5b in the Repair of Hair Cells in Sea Anemones
}

\author{
Glen M. Watson, Erin M. Graugnard, and Patricia Mire \\ Department of Biology, University of Louisiana at Lafayette, Lafayette, LA 70504-2451, USA
}

Received: 30 August 2006; Accepted: 12 January 2007; Online publication: 27 February 2007

\begin{abstract}
The subcellular processes involved in repair of hair cells are not well understood. Sea anemones repair hair bundle mechanoreceptors on their tentacles after severe trauma caused by 1-h exposure to calcium-depleted seawater. Repair is dependent on the synthesis and secretion of large protein complexes named "repair proteins." A cDNA library on traumatized anemone tissue was probed using polyclonal antibodies raised to a specific chromatographic fraction of the repair protein mixture. An ADPribosylation factor-like protein, Arl-5b, was identified. The amino acid sequence of the Arl-5b protein in sea anemones is similar to that among several model vertebrates and humans. A polyclonal antibody raised to a peptide of the anemone Arl-5b labels some but not all hair bundles in healthy control animals. The abundance of labeled hair bundles significantly increases above healthy controls after trauma and continuing through the first hour of recovery. Dilute anti-Arl-5b blocks the spontaneous repair of hair bundle mechanoreceptors, suggesting that Arl-5b acts on the extracellular face of the plasma membrane. Immunoelectron microscopy indicates that Arl-5b is located along the length of stereocilia including sites in the vicinity of tip links. We propose that Arl-5b is involved in installing replacement linkages into damaged hair bundle mechanoreceptors.
\end{abstract}

Correspondence to: Glen M. Watson - Department of Biology · University of Louisiana at Lafayette - Lafayette, LA 70504-2451, USA. Telephone: +1-337-4825667; fax: +1-337-4825660; email: gmw5722@louisiana.edu
Keywords: ADP-ribosylation factor-like protein, ADP-ribosyltransferase

\section{INTRODUCTION}

Although it is widely accepted that hair cells are capable of at least limited repair, the subcellular processes involved are not well understood. In at least some instances, it appears that sublethally damaged hair cells replace the entire hair bundle mechanoreceptor apparatus (Sobkowicz et al. 1992; Baird et al. 2000). In other instances, the existing hair bundle is repaired. Repair of existing hair bundle mechanoreceptors includes (1) reorganizing the actin cytoskeleton within stereocilia and (2) repairing or replacing damaged linkages that normally interconnect stereocilia. With respect to actin, one example involves damage to hair cells caused by ejection of strong jets of buffer. This over stimulation induces a softening of the hair bundle that spontaneously resolves in a manner thought to result from a restructuring of the cytoskeleton within the stereocilia (Duncan et al. 1995; Duncan and Saunders 2000). Noise trauma increases expression of cdc- 42 in the sensory epithelium of the chick cochlea (Gong et al. 1996). This finding is significant because cdc- 42 is involved in regulating actin dynamics (Hall 1998). Likewise the actin-binding protein, WDR1, is upregulated after noise trauma (Oh et al. 2002). WDR1 may interact with $\mathrm{ADF}$ to enhance actin turnover in the sensory epithelium after noise trauma $(\mathrm{Oh}$ et al. 2002). In mammals, self-repair of hair cells occurring within $24-48 \mathrm{~h}$ of moderate noise trauma may be linked to a regular turnover of the actin cytoskeleton within stereocilia (Rzadzinska et al. 2004). With respect to linkages, noise trauma can damage or 
destroy linkages interconnecting stereocilia (Clark and Pickles 1996; Pickles et al. 1987; Husbands et al. 1999). In chickens, noise damaged hair cells replace tip links within 24-48 h (Husbands et al. 1999). In the laboratory, hair cells from chickens lose mechanosensitivity after brief exposure to calcium-depleted buffers. Such exposure may mimic noise trauma, at least in part, because calcium-depleted buffers damage or destroy linkages interconnecting stereocilia (Assad et al. 1991; Zhao et al. 1996; Goodyear and Richardson 1999). Within $12 \mathrm{~h}$, the traumatized hair cells replace missing tip links and recover mechanosensitivity (Zhao et al. 1996). In cochlear cultures from mice, exposure to calcium-depleted buffers abolishes uptake of FM1-43 into the hair cells. Within $24 \mathrm{~h}$, uptake of FM1-43 recovers to control levels (Gale et al. 2001). FM1-43 is thought to enter hair cells through functional transduction channels (i.e., those associated with intact gating springs) (Meyers et al. 2003). In vertebrate animals, repair is limited to hair cells having suffered mild to moderate damage. Severe damage to hair cells results in cell death.

In contrast, hair cells in sea anemones repair after severe damage. Sea anemones employ hair bundle mechanoreceptors located on their tentacles to detect the swimming movements of planktonic prey (Watson and Hessinger 1989; Watson and Mire 2004). Hair bundle mechanoreceptors are derived from a multicellular complex in which two to four supporting cells surround a single sensory neuron (Fig. 1). The sensory neuron contributes a kinocilium and six large-diameter stereocilia to the hair bundle. The supporting cells contribute 200-300 small-diameter stereocilia to the hair bundle. Linkages typical of vertebrate hair cells including tip links, lateral links, and basal linkages interconnect the stereocilia (Watson et al. 1997). Because the majority of stereocilia originate from the supporting cells, they carry the majority of the transduction current (Mire and Watson 1997). Such "supporting cells" would be more aptly named "hair cells" despite the fact that the term supporting cell has historical precedence. We will refer to such cells as hair cells throughout the remainder of the paper. In the laboratory, vibration sensitivity is evaluated at the organismal level using a novel bioassay based on counting nematocysts discharged into test probes in the presence of vibrations at appropriate frequencies (Watson and Hudson 1994). Nematocysts are "stinging organelles" employed to capture prey (Mariscal 1974). After exposing anemones to calcium-depleted seawater for $1 \mathrm{~h}$, hair bundles are profoundly disorganized and vibration sensitivity is abolished. Within $4 \mathrm{~h}$ after returning the sea anemones to calcium-containing seawater, normal morphology of the hair bundles and normal vibration sensitivity are restored (Watson et al.
1998). The repair process involves a significant loss of F-actin from the stereocilia, followed by a recovery of F-actin (Watson and Mire 2001). Furthermore, recovery is dependent on large, secreted protein complexes named "repair proteins." If traumatized anemones are exposed to fraction $\beta$, a chromatographic fraction of the repair protein mixture, a complete recovery of vibration sensitivity occurs at the organismal level within 7-8 $\min$ (Watson et al. 1998). However, a full recovery of normal morphology and F-actin content of the hair bundle lags behind, requiring $45 \mathrm{~min}$ of exposure to fraction $\beta$. Likewise, a full recovery of the mechanoelectric current requires 45 min of exposure to fraction $\beta$. Hence, fraction $\beta$ restores vibration sensitivity at the organismal level before full functionality is restored to each hair bundle (Watson and Mire 2001). According to the working model, fraction $\beta$ includes replacement linkages of all types and accessory

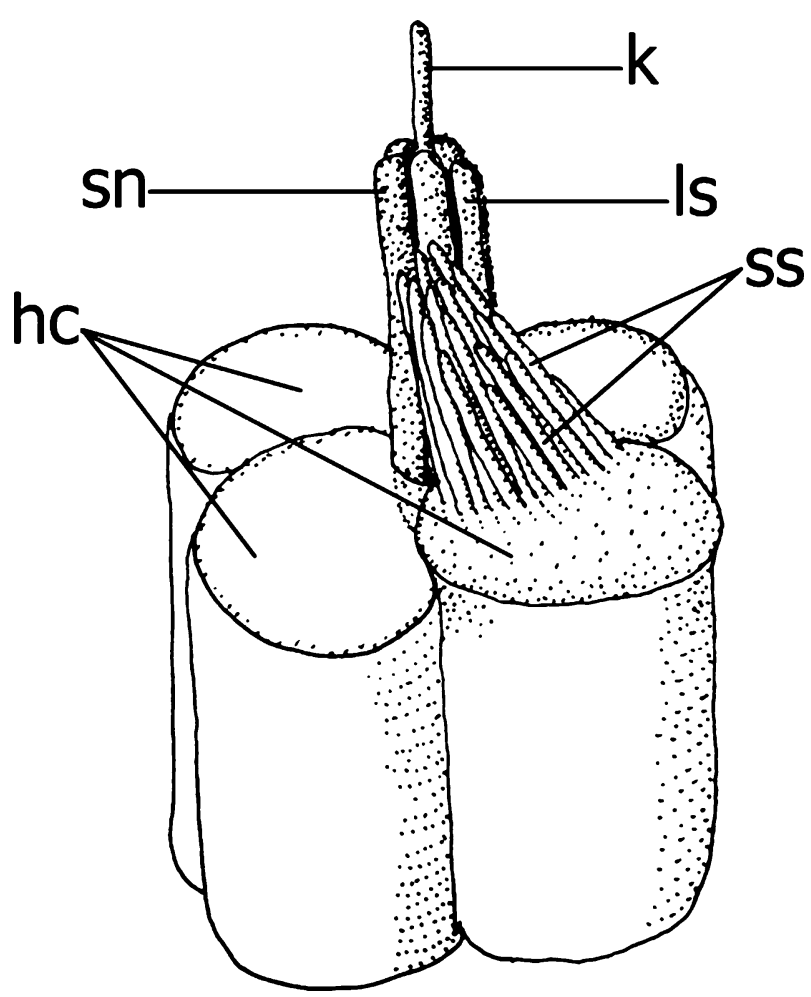

FIG. 1. Diagram of a hair bundle mechanoreceptor on an anemone tentacle. The hair bundle mechanoreceptor originates from a multicellular complex consisting of a single sensory neuron (sn) surrounded by four supporting cells: "hair cells" (hc). The hair bundle is radially symmetric with a kinocilium $(k)$ at the center surrounded by six large-diameter stereocilia (Is). The kinocilium and large-diameter stereocilia originate from the sensory neuron. Numerous small-diameter stereocilia (ss) from the hair cells converge onto the large-diameter stereocilia. To allow visualization of the sensory neuron, small-diameter stereocilia were drawn from only one of the four hair cells. In an actual hair bundle mechanoreceptor, small-diameter stereocilia from all four of the hair cells would converge onto the centrally located, large-diameter stereocilia. 
proteins that participate in installing the replacement linkages (Watson et al. 1998). There is a great deal yet to be learned about the biochemical mechanisms by which these and other hair cells self-repair. In this paper, we provide evidence that Arl-5b, an ADP-ribosylation factorlike protein, is involved as an extracellular factor in the repair of hair cells in sea anemones.

\section{MATERIALS AND METHODS}

Animal maintenance. Sea anemones, Haliplanella luciae, were maintained in shallow glass dishes containing natural seawater at $32 \%$ and $16^{\circ} \mathrm{C}$. The animals were fed to repletion twice weekly using freshly hatched Artemia nauplii (Minasian and Mariscal 1979). By these simple methods we have maintained continuous cultures of $H$. luciae for more than 20 years.

Molecular biology. A lambda-based cDNA library was constructed using the Stratagene ZAP-cDNA Gigapack III Gold Cloning Kit (La Jolla, CA, USA) according to the manufacturer's instructions. The mRNA was isolated from anemones that had been traumatized in calcium-depleted seawater for $10 \mathrm{~min}$ followed by a 30-min recovery in calcium-containing seawater. This treatment regime was used after pilot studies using the transcription inhibitor, actinomycin-D, or the translation inhibitor, cycloheximide, indicated that a $30-\mathrm{min}$ recovery permitted ample transcription but little translation, (i.e., whereas at $30 \mathrm{~min}$ recovery actinomycin-D was no longer effective in delaying the recovery of vibration sensitivity relative to untreated controls, cycloheximide was maximally effective in delaying the recovery of vibration sensitivity). We reasoned that animals killed at this time point might have an ideal complement of mRNA. The cDNA library from the $H$. luciae mRNA was cloned into the lambda phage vector, UNI-ZAP-XR (Stratagene). The library was used to infect Escherichia coli (XL1-Blue MRF' strain, Stratagene) according to the manufacturer's instructions. The library was screened for clones that were immunopositive to a polyclonal antibody raised to deglycosylated fraction $\beta$ (Watson and VenableThibodeaux 2000) using Stratagene's picoblue screening kit. Bacteria were grown at $42^{\circ} \mathrm{C}$ until plaques were just visible. Plates were overlain with nitrocellulose paper that had previously been soaked in $10 \mathrm{mM}$ of isopropyl- $\beta$-D-thiogalactopyranoside and then incubated overnight at $37^{\circ} \mathrm{C}$. The fraction $\beta$ antiserum had been pretreated with $E$. coli per phage lysate absorbed onto nitrocellulose paper to extract antibodies that react to $E$. coli or phage antigens. The filters were washed in TBST $(20 \mathrm{mM}$ Tris- $\mathrm{HCl}, 150 \mathrm{mM} \mathrm{NaCl}$, and $0.05 \%$ Tween 20, pH 7.5), blocked in $1 \%$ bovine serum albumin (BSA) in TBS (20 mM Tris- $\mathrm{HCl}$ and $150 \mathrm{mM}$
$\mathrm{NaCl}, \mathrm{pH} 7.5)$, and then incubated in a $1 / 3,000$ dilution of fraction $\beta$ antiserum in BSA/TBS at $4^{\circ} \mathrm{C}$ overnight. The filters were washed in TBST and then incubated in a 1/1,600 dilution of anti-rabbit IgG-alkaline phosphatase for $1 \mathrm{~h}$ at room temperature. Color development in $\mathrm{BCIP} / \mathrm{NBT}$ showed bacteria producing fusion peptides. Single-stranded DNA was isolated from immunopositive clones according to the manufacturers' instructions and then sequenced using the ABI-3100 sequencer and Big-Dye 3.1 terminator dye set (Applied Biosystems, Foster City, CA, USA). The data were analyzed using Lasergene software (DNASTAR, Madison, WI, USA).

Bioassay for evaluating vibration sensitivity. Vibration sensitivity was assayed using a modification of methods described previously (Watson and Hudson 1994). Specimens were allowed to attach to small petri dishes immersed in flat glass culture dishes filled with seawater. The petri dishes were transferred to larger petri dishes in which they could be completely immersed. Two-centimeter segments of fishing line were coated with gelatin to a thickness of approximately $200 \mu \mathrm{m}$. Uncoated ends of the fishing line were held by fine forceps and lowered into the water near a glass capillary tube attached to a piezo disk induced to vibrate by a Telulex SG-100 digital function generator equipped with a frequency counter (Telulex, Inc., Mountain View, CA, USA). The glass capillary tube vibrated at $55 \mathrm{~Hz}$, a frequency previously shown to elicit strong discharge of nematocysts. The test probe was allowed to hydrate for $5 \mathrm{~s}$ before it was moved into contact with the tentacles of an anemone. The probe was then swiftly withdrawn in a direction that minimized the chance for additional tentacles to touch the probe. Test probes were fixed in $2.5 \%$ glutaraldehyde. A total of eight test probes was obtained, one each from eight different animals. Test probes were examined by phase contrast microscopy at $\times 400$ magnification. Discharged microbasic $p$-mastigophore nematocysts were counted for a single representative field of view. Data points indicate the mean number of nematocysts counted per field of view plus the standard error of the mean. Statistical analyses were performed using CSS Statistica software (Statsoft, Tulsa, OK, USA). Graphs were prepared using Origin 5.0 (Microcal, Northampton, MA, USA).

Production of fraction $\beta$. Fraction beta was collected from anemones as described previously (Watson and Mire 2001).

Immunocytochemistry. Anemones were anesthetized in KSW (in millimolar concentrations: $\mathrm{NaCl} 323$, $\mathrm{MgSO}_{4}$ 26, $\mathrm{MgCl}_{2}$ 24, KCl $100, \mathrm{CaCl}_{2}$ 12, and $\mathrm{NaHCO}_{3} 2$ ) for $1.5 \mathrm{~h}$ and then traumatized or not 
(healthy controls) in calcium-depleted KSW (in millimolar concentrations: $\mathrm{NaCl} 347, \mathrm{MgSO}_{4} 26, \mathrm{MgCl}_{2}$ 24, $\mathrm{KCl} 100, \mathrm{CaCl}_{2}$ 0, ethylene glycol bis (2-aminoethyl ether)- $N, N, N^{\prime}, N^{\prime}$-tetraacetic acid (EGTA) 8 , and $\mathrm{NaHCO}_{3} 2$ ) for $1 \mathrm{~h}$. The animals were returned to KSW and then fixed using $1.0 \%$ glutaraldehyde (or $0.05 \%$ glutaraldehyde) and $4 \%$ paraformaldehyde prepared in Millonig's buffer augmented with 3\% $\mathrm{NaCl}$ for $30 \mathrm{~min}$ at room temperature. Specimens were rinsed in phosphate-buffered saline (PBS) and then blocked in 3\% BSA in PBS (BSA/PBS). Specimens were incubated in a $1 / 200$ dilution of anti-Arl$5 \mathrm{~b}$ (or preimmune serum for controls) in BSA/PBS for $30 \mathrm{~min}$ (or overnight at $4^{\circ} \mathrm{C}$ ), rinsed in PBS, blocked in BSA/PBS, and then incubated in antirabbit IgG conjugated to rhodamine in BSA/PBS for 30-60 min. In some experiments, specimens were labeled in phalloidin-Oregon Green (Molecular Probes). The tissue was rinsed in PBS and then transferred to $100 \mu \mathrm{M}$ of citric acid in PBS to retard bleaching. Excised tentacles were prepared as wet mounts and then imaged using a LOMO Lumam epifluorescent microscope (model RP011-T, LOMO America, Prospect Heights, IL, USA). The principal objectives used were a $40 \times$ fluorite $(\mathrm{na}=0.85)$ and a $100 \times$ oil-immersion fluorite $($ na $=1.30)$. Images were obtained using a STL-11000 SBIG cooled CCD camera (SBIG, Santa Barbara, CA, USA) controlled by MaximDL software (Diffraction Limited, Ontario, Canada). Immunoelectron microscopy was performed as follows. Specimens were fixed in $0.05 \%$ glutaraldehyde and $4 \%$ paraformaldehyde in Millonig's buffer containing $3 \% \mathrm{NaCl}$ for $30 \mathrm{~min}$. Each specimen was bisected for subsequent exposure to Arl-5b antiserum (experimentals) or preimmune serum (controls). Remaining free aldehydes of glutaraldehyde were blocked by immersing the tissue in $0.1 \mathrm{M}$ of glycine in PBS for $5 \mathrm{~min}$. The tissue was rinsed in PBS for $5 \mathrm{~min}$ and then incubated in BSA/PBS for $30 \mathrm{~min}$ to block nonspecific binding sites. Experimental tissue was incubated overnight in a 1/200 dilution of antiArl-5b in BSA/PBS at $4^{\circ} \mathrm{C}$. Control tissue was incubated overnight in a 1/200 dilution of preimmune serum in $\mathrm{BSA} / \mathrm{PBS}$ at $4^{\circ} \mathrm{C}$. The tissue was rinsed in PBS, blocked in BSA/PBS, and then incubated in antirabbit IgG conjugated to colloidal gold (Sigma) diluted $1 / 25$ in BSA/PBS for $1 \mathrm{~h}$ at room temperature. The tissue was rinsed in PBS for $30 \mathrm{~min}$ and then fixed in $1 \%$ glutaraldehyde and $4 \%$ paraformaldehyde in Millonig's buffer for $1 \mathrm{~h}$. The tissue was rinsed in PBS, then dehydrated in acetone and embedded in Spurr resin. Thin sections were osmicated for $10 \mathrm{~min}$ on drops of $0.04 \% \mathrm{OsO}_{4}$ and then counterstained in $1 \%$ uranyl acetate for $1 \mathrm{~h}$ and a triple lead stain for 5 min. Sections were viewed using a Hitachi 7000 TEM operating at $75 \mathrm{kV}$.
Sodium dodecyl sulfate polyacrylamide gel electrophoresis (SDS-PAGE) was performed using BioRad Mini-Protean II apparatus at a constant voltage of $200 \mathrm{~V}$ (BioRad Laboratories, Hercules, CA, USA). Western Blots on nitrocellulose membranes were obtained at $6 \mathrm{~V}$ for 30 min from $15 \%$ acrylamide gels using the Genie Blotter (Idea Scientific, Minneapolis, MN, USA). Blots were probed overnight at $4^{\circ} \mathrm{C}$ using a 1/1,000 dilution of Arl-5b antiserum (or preimmune serum for control lanes) in 3\% BSA in PBS. The secondary antibody conjugated to horseradish peroxidase was diluted $1 / 1,000$ in $3 \%$ BSA in PBS for $1 \mathrm{~h}$ at room temperature before color development using chloronaphthol.

\section{RESULTS}

ARL-5b in anemones is similar to Arl-5b in model vertebrates

The homolog of Arl-5b (ADP-ribosylation factor-like protein-5b) in the anemone, H. luciae, was isolated from a cDNA library generated to traumatized anemones. A polyclonal antibody to fraction $\beta$ was used to screen candidate clones for genes coding for polypeptides in fraction $\beta$. The estimated mass of the Arl-5b polypeptide in humans, frogs, and zebrafish is approximately $20.4 \mathrm{kDa}$. Among these vertebrates, the sequence is approximately $95 \%$ identical (Fig. 2). Translation of the sequenced open reading frame of the H. luciae homolog is shown in Figure 2. Assuming that the $H$. luciae Arl-5b is the same size as that in vertebrate animals, $94 \%$ of the protein is sequenced with the missing $6 \%$ of the protein at the $\mathrm{N}$ terminus. For the known portion of the protein, the $H$. luciae homolog is $76-78 \%$ identical to those in model vertebrates. Nearly half of amino acid substitutions in the $H$. luciae protein relative to the consensus sequence are for similar types of amino acids. Thus, the H. luciae homolog of Arl-5b is approximately $87 \%$ "similar" to those of model vertebrates. The $H$. luciae homolog of Arl-5b also was compared with the fully sequenced homolog from the model anemone, Nematostella vectensis (Darling et al. 2005). The two anemone homologs (H. luciae and N. vectensis) of Arl$5 \mathrm{~b}$ were $90 \%$ identical to each other. The anemone homologs of Arl-5b were more negatively charged than those in the vertebrate animals. Interestingly, amino acid substitutions in the two anemone sequences relative to the consensus sequence derived from all five sequences tended to occur at similar loci (Fig. 2). To analyze the occurrence of structural motifs in the $H$. luciae sequence, we constructed a chimera anemone sequence in which the first 15 residues were borrowed from Nematostella. Several motifs are identified in the anemone chimera primary sequence 


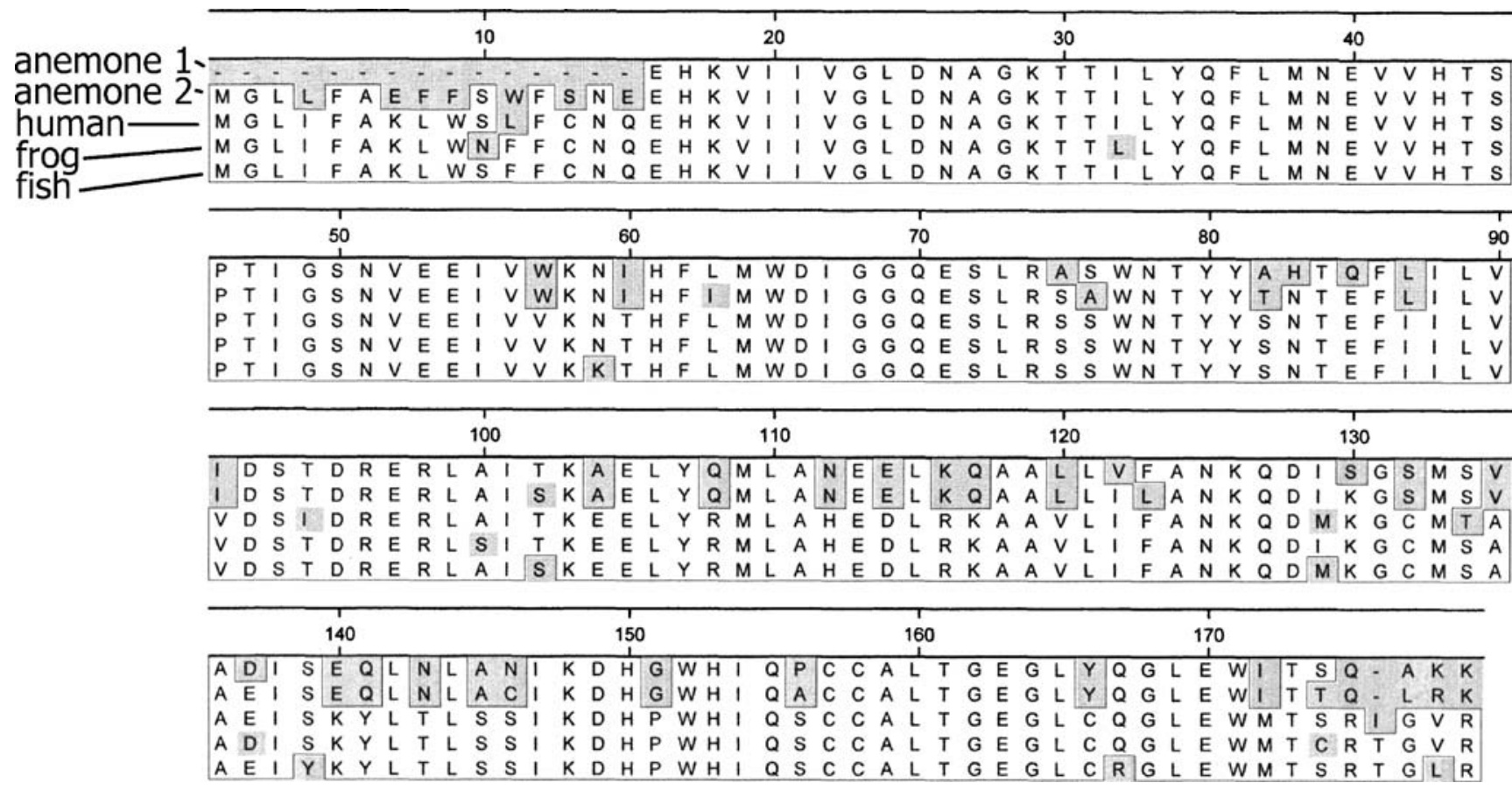

FIG. 2. Sequence alignment of residues for Arl-5b. Homologs of the sequenced proteins are shown for (anemone 1) H. luciae (partial sequence, top row), (anemone 2) N. vectensis (second row, dv082782), (human) Homo sapiens (third row, CAH70494.1), (frog) Xenopus tropicalis (fourth row, AAH75510.1), and (fish) Danio rerio (bottom row, AAH52766.1). Sequences are referenced by their accession numbers (see the protein database http://www.ncbi.nlm.nih.gov/entrez/query.fcgi). Residues that differ from the consensus are shaded.

data, including putative myristoylation sites, a GTPbinding site, putative regulatory sites by casein kinase II and by protein kinase $\mathrm{C}$, and a signature motif for members of the ADP-ribosylation factor family (Fig. 3).

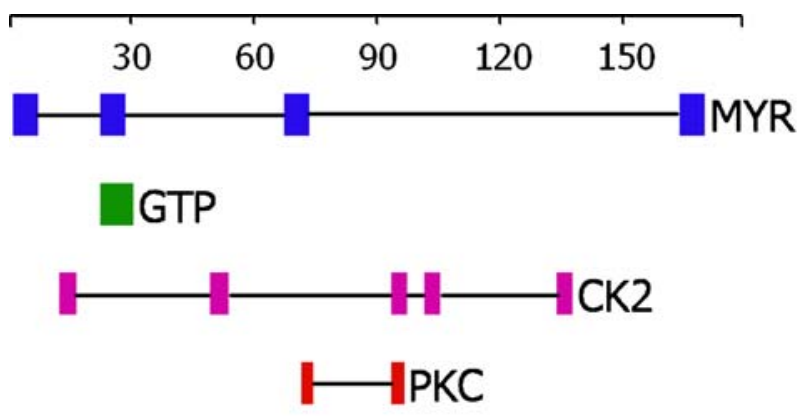

ARF

FIG. 3. Structural motifs identified from a chimera sequence of Arl$5 \mathrm{~b}$ from anemones based on the $\mathrm{N}$-terminal 15 residues from Nematostella and the remaining 163 residues from Haliplanella. The protein sequence was analyzed using the Prosite database accessed using the ExPASy proteomics server of the Swiss Institute of Bioinformatics (http://ca.expasy.org). The motifs, aligned adjacent to the primary sequence, are as follows: putative myristoylation (MYR), GTP binding (GTP), phosphorylation by casein kinase 2 (CK2), phosphorylation by protein kinase C (PKC), and an Arf family domain (ARF).
Immunobiology

A polyclonal antibody was generated from the sequence data for the $H$. luciae Arl-5b to a peptide constructed from residues 98-117 (Alpha Diagnostics International, San Antonio, TX, USA). Small aliquots of fraction $\beta$ were applied to nitrocellulose paper and then probed with either the Arl-5b antiserum or the preimmune serum (Fig. 4A). Whereas the Arl-5b antiserum recognized fraction $\beta$, the preimmune serum did not (Fig. 4A). A Western blot to repair protein showed an immunoreactive band to the Arl$5 \mathrm{~b}$ antiserum at approximately $18 \mathrm{kDa}$ (Fig. 4C) that was not detected in the preimmune lane (Fig. 4D). The predicted mass of the anemone chimera Arl-5b protein is $20.2 \mathrm{kDa}$. Immunocytochemistry of healthy control animals showed that anti-Arl-5b labeled some (Fig. 5A), but not all hair bundles. The preimmune serum weakly labeled or did not label the hair bundles (Fig. 5B). To test the possibility that Arl-5b is upregulated in damaged hair cells, a time course study was performed in which anemones were traumatized in calcium-depleted seawater for $1 \mathrm{~h}$ or not (healthy controls) and then fixed at increments during the 4-h spontaneous recovery. Quantitative data were obtained for four replicate experiments wherein the number of immunopositive hair bundles was counted per unit area. Compared to healthy 


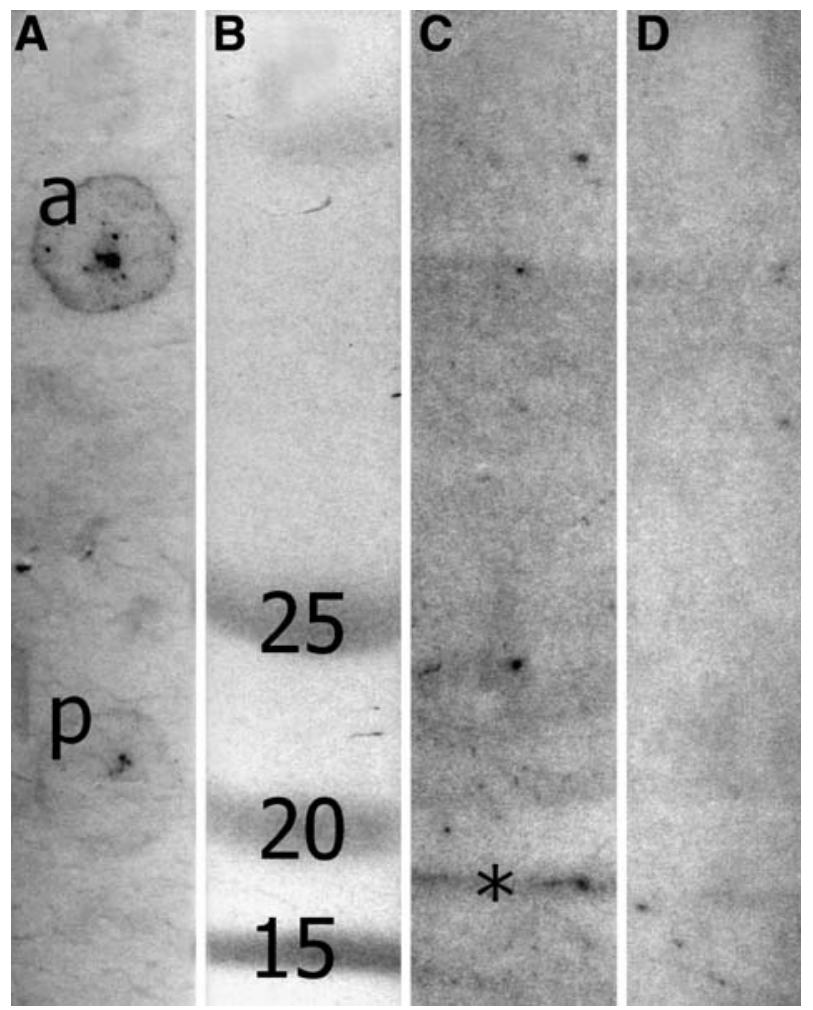

FIG. 4. Protein blots of Arl-5b. A Dot blot of fraction $\beta$ probed with (a) a polyclonal antibody generated to a deduced peptide of Arl-5b or (p) preimmune serum. B-D Western blot of "repair protein" from anemones. The lanes are as follows: B protein standards with the mass in kilodalton, $\mathbf{C}$ Arl-5b antiserum. An immunopositive band (asterisk) is located between the standards at 15 and $20 \mathrm{kDa}$; and D preimmune serum. The blots were photographed together. Contrast was adjusted on the master image so that differences between the treatments would be faithfully reproduced.

controls, immunopositive hair bundles significantly increased in abundance after removing the animals from the calcium-depleted seawater and continuing through the first hour after trauma. Thereafter, the abundance of immunopositive hair bundles gradually decreased (Fig. 6).

Anemones were traumatized in calcium-depleted seawater for $1 \mathrm{~h}$ and then allowed to spontaneously recover vibration sensitivity in calcium-containing seawater over a 7-h period. The seawater was augmented by Arl-5b antiserum for the experimental animals and preimmune serum for the control animals. In each case, the serum was diluted in seawater to 1 part in 500 . Whereas the control animals recovered vibration sensitivity in $4 \mathrm{~h}$, as has been shown previously in seawater alone (Watson et al. 1998), the experimental animals incubated in the presence of the Arl-5b antiserum failed to recover normal vibration sensitivity even after $7 \mathrm{~h}$ (Fig. 7). The morphology of the recovering hair bundles was investigated using phalloidin staining to label the stereocilia. In the presence of the Arl-5b antiserum, large-diameter stereocilia were splayed at $1 \mathrm{~h}$ posttrauma and appeared to have few, if any, small-diameter stereocilia converging onto the large-diameter stereocilia (i.e., the splayed hair bundles consisted of approximately six stereocilia) (Fig. $8 \mathrm{~A})$. In contrast, traumatized controls incubated in the preimmune serum and allowed to recover for the same length of time showed a modest reorganization of the large-diameter and small-diameter stereocilia to form a loosely organized bundle (i.e., the hair bundles consisted of many more than six stereocilia, indicating the participation of large-diameter and small-diameter stereocilia) (Fig. 8B).

Immunoelectron microscopy was performed on healthy control animals. Gold particles were observed almost exclusively on stereocilia of hair bundles where they commonly occurred distally in the membrane of stereocilia in the region of tip links (Figs. 9A-C). Of 988 gold particles counted across a comparable area of tissue, 99 gold particles occurred in tissue exposed to preimmune serum and 887 occurred in tissue exposed to anti-Arl-5b.

ADP-ribosylation factors can activate ADP-ribosyltransferases. To test the possibility that ADP-ribosyltransferases might be involved in the repair process, $\beta$-NAD and fraction $\beta$ were exogenously supplied to animals traumatized by $1-\mathrm{h}$ exposure to calciumdepleted seawater. $\beta$-NAD is the substrate for ADPribosyltransferases. The ADP-ribosyltransferase cleaves $\beta-N A D$ into nicotinamide and ADP-ribose; the latter is transferred to the substrate protein. Vibration sensitivity was tested 2 min after adding $\beta$-NAD and fraction $\beta$. A 2-min exposure to fraction $\beta$ alone failed to increase discharge above traumatized controls that received no treatment after trauma (Fig. 10). On the other hand, 2-min exposure to fraction $\beta$ in the presence of $\beta$-NAD restored discharge to levels nearly identical to healthy controls at $10^{-4} \mathrm{M}$ of $\beta$-NAD and statistically indistinguishable from healthy controls down to $10^{-8} \mathrm{M}$ of $\beta$-NAD (Fig. 10).

\section{DISCUSSION}

Arl-5b is identified in a cDNA library constructed using traumatized anemones

Arl-5b is a member of the Arf family (Kahn et al. 2006). Arfs are ADP-ribosylation factors, small GTP-binding proteins originally named as essential cofactors in pathways involving ADP-ribosylation of substrates. GTP binding converts the Arfs from cytoplasmic proteins to membrane-associated proteins; the latter being the active form. At membranes, Arfs interact with a large number of effectors, including coatforming complexes, adapters, and lipid modifying enzymes. GTP binding is facilitated by Arf guanine 

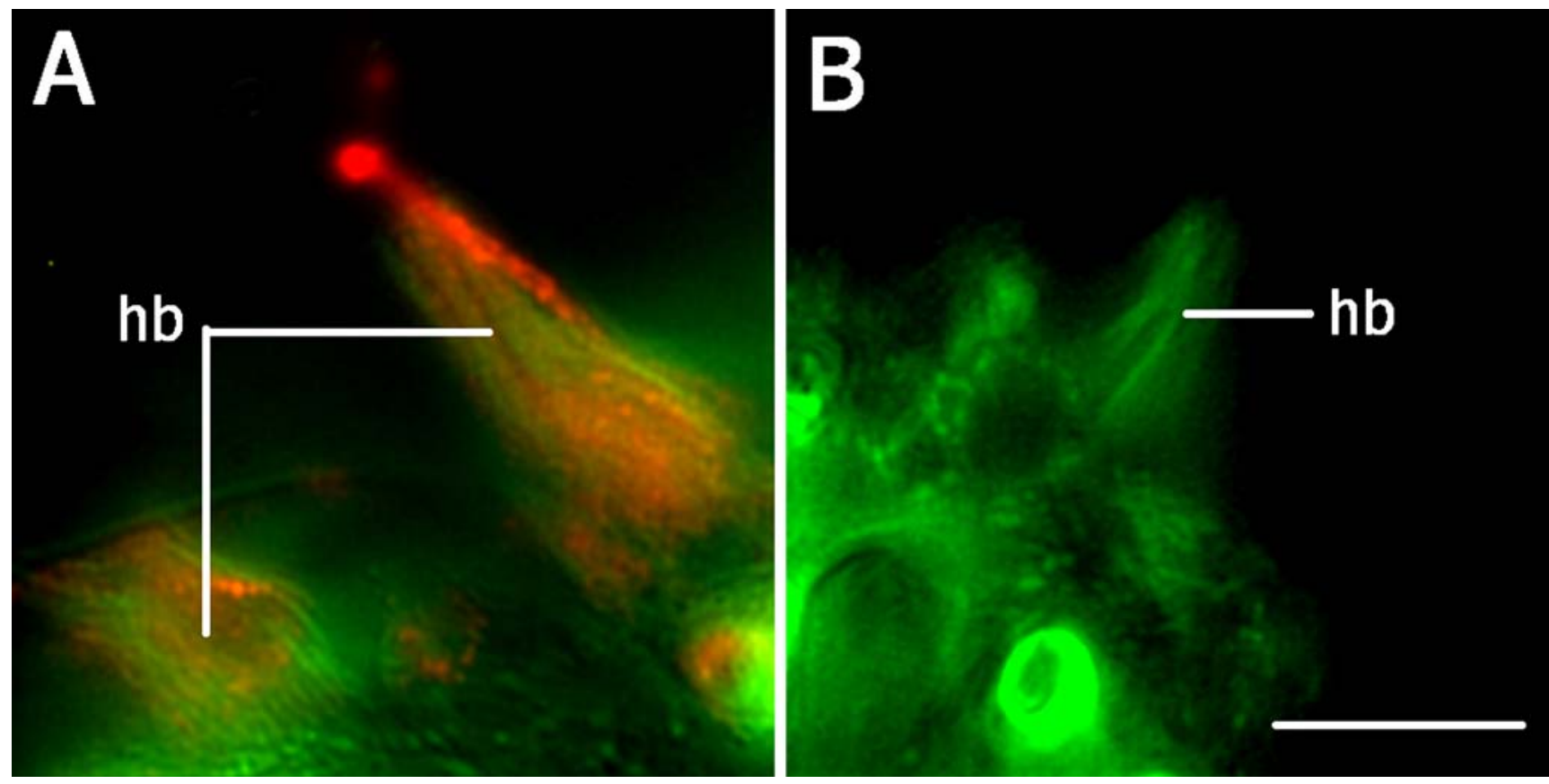

FIG. 5. Immunocytochemistry of Arl-5b performed on healthy anemone tentacles. This specimen was double-labeled for immunocytochemistry (red) and for F-actin using phalloidin (green). Healthy control animals were fixed and bisected. One half of the specimen was processed for immunocytochemistry to anti-Arl-5b (subpanel A) and the other half was processed for immunocytochemistry using the preimmune serum (subpanel B). A Two immunolabeled hair bundles (hb) are shown. B In the specimen exposed to preimmune serum, no antibody labeling is visible. A single hair bundle ( $\mathrm{hb}$ ) is shown. The images used in subpanels $\mathbf{A}$ and $\mathbf{B}$ were collected using the same exposure time and contrasted using the same settings. The images were deconvolved using a Lucy-Richardson algorithm to sharpen details. Scale bar $=10 \mu \mathrm{m}$.

nucleotide exchange factors. GTP hydrolysis is stimulated by Arf GTPase-activating proteins. In humans the Arf family comprises 29 members, including Arls (Kahn et al. 2006). Arls are "Arf-like proteins" in

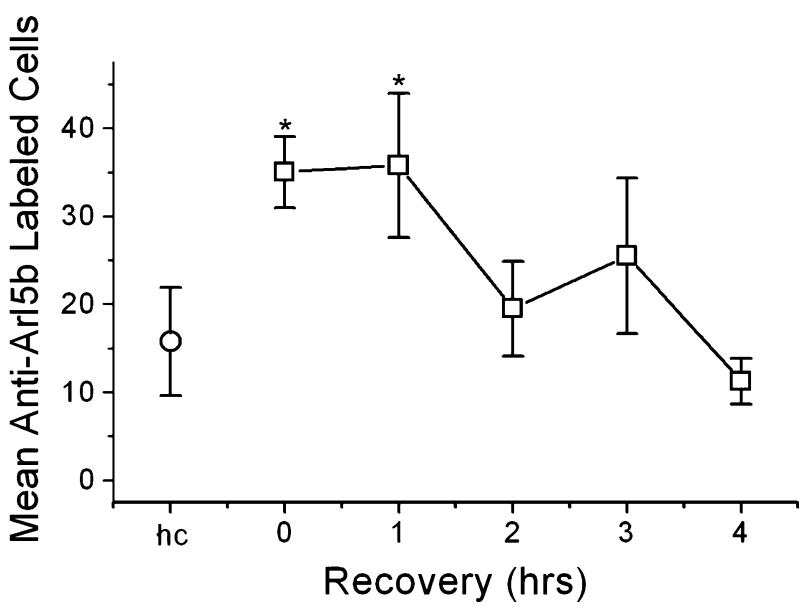

FIG. 6. Quantitation of immunopositive hair bundles during the spontaneous recovery to trauma. Anemones were traumatized in calcium-depleted seawater for $1 \mathrm{~h}$ (or not for healthy controls, hc), and then were transferred to calcium-containing seawater where they remained for specified intervals before they were fixed and processed for immunocytochemistry to Arl-5b. Immunopositive hair bundles were counted within a grid placed on the computer monitor. Data points indicate the mean number of hair bundles \pm SEM for four replicate experiments. Data differing significantly from healthy controls are indicated by asterisks. terms of having sequences related to Arfs. However, Arls are considered to represent a structurally and functionally diverse group with very little known about their functions.

Arl-5b is a component of fraction $\beta$ Immunoblots confirmed that fraction $\beta$ includes Arl-5b. Because fraction $\beta$ is purified from the mucus ring found at the base of the anemones having recently recovered from trauma to their hair bundles, it appears that Arl-5b is externalized during the repair process.

Evidence that Arl-5b is involved in repair of hair cells Immunolabeling of hair cells by anti-Arl-5B significantly increased in abundance after trauma in calciumdepleted seawater. Hence, increased expression or secretion of Arl-5b follows trauma to anemone hair bundles.

The case for an ectoprotein mediated repair process Adding Arl-5b antiserum, but not preimmune serum, to the seawater containing the anemones blocked the recovery of vibration sensitivity. Such inhibition confirms that Arl-5b is involved in the repair process. Furthermore, these data suggest that Arl-5b functions extracellularly.

Speculation about Arl-5b as an ectoprotein Some predictions about the biology of Arl-5b emerge from its primary sequence. Arl-5b may be myristoylated, most likely at the amino terminus. Myristate tails serve to reversibly anchor proteins at the membrane. 


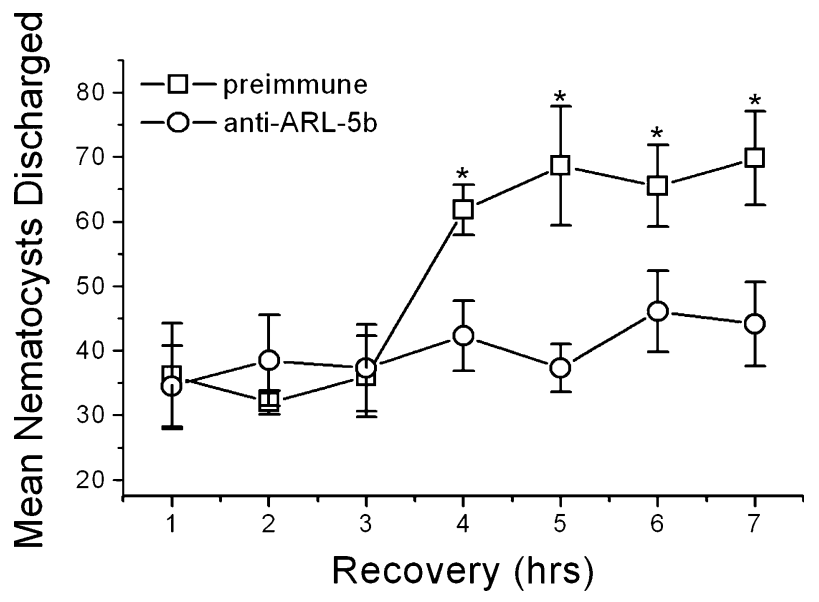

FIG. 7. Effects of Arl-5b antiserum on the recovery of vibrationdependent discharge of nematocysts. Anemones were traumatized in calcium-depleted seawater for $1 \mathrm{~h}$, and then were transferred to calcium-containing seawater where they remained for specified intervals before they were touched with gelatin-coated, test probes in the presence of vibrations at $55 \mathrm{~Hz}$. Experimental animals (circles) were incubated in Arl-5b antiserum diluted in seawater to 1/500. Control animals (squares) were incubated in preimmune serum from the same rabbit diluted to $1 / 500$. Microbasic p-mastigophore nematocysts discharged into the gelatin coat were counted for a representative field of view at $\times 400$ magnification. Data points indicate the mean number of nematocysts \pm SEM counted for eight replicate test probes, each touched to a different anemone. Statistical comparisons were made using post hoc tests after the ANOVA wherein experimental groups were compared to control groups for the same time point (e.g., experiment $1 \mathrm{~h}$ recovery vs control $1 \mathrm{~h}$ recovery). Significant differences are indicated by asterisks.
The ADP-ribosylation factor, Arf1, cycles between a membrane bound, active form and a soluble, inactive form according to whether GTP or GDP is bound. In its active form, Arf1 (with GTP bound) exposes its $\mathrm{N}$ myristoyl group. The exposed myristate tail targets Arfl to the membrane (Goldberg 1998). Secreted acylated proteins are known to occur and include such paracrine factors as members of the hedgehog family (Ingham and McMahon 2001). Full inductive activity of sonic hedgehog in the rat brain requires the N-terminal fatty acylation of the protein (Kohtz et al. 2001). Thus, Arl-5b may be myristoylated. If so, the presence of the myristate tail need not interfere with secretion. The myristate tail may be involved in targeting Arl-5b to membranes.

Putative functions for Arl-5b Many questions emerge. At the forefront of these questions is what precisely is Arl-5b doing with respect to repair? The time course experiments based on immunocytochemistry suggest that Arl-5b functions early in the repair process. At 1-h recovery and in the presence of the Arl-5b antiserum, large-diameter stereocilia from the sensory neuron were markedly splayed compared to those from animals incubated in the preimmune serum for the same length of time. Furthermore, few, if any, small-diameter stereocilia were associated with the large-diameter stereocilia in specimens exposed to Arl-5b antiserum. Thus, it appears that Arl-5b somehow is involved in assisting the stereocilia of damaged hair bundles to reorganize into a bundle. Extracellular linkages provide a critical function in maintaining the structural integrity of hair bundles. In the absence of such linkages,
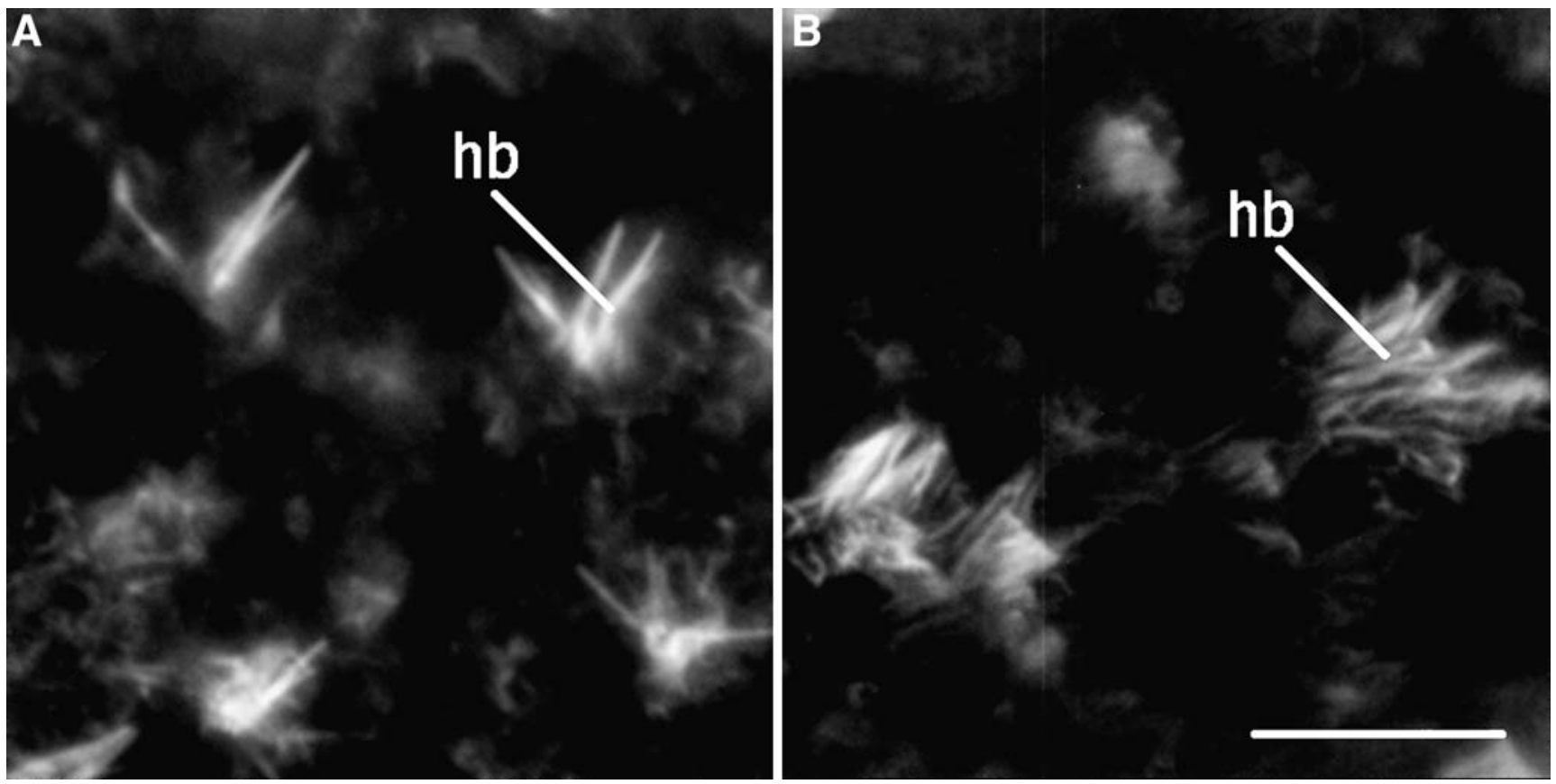


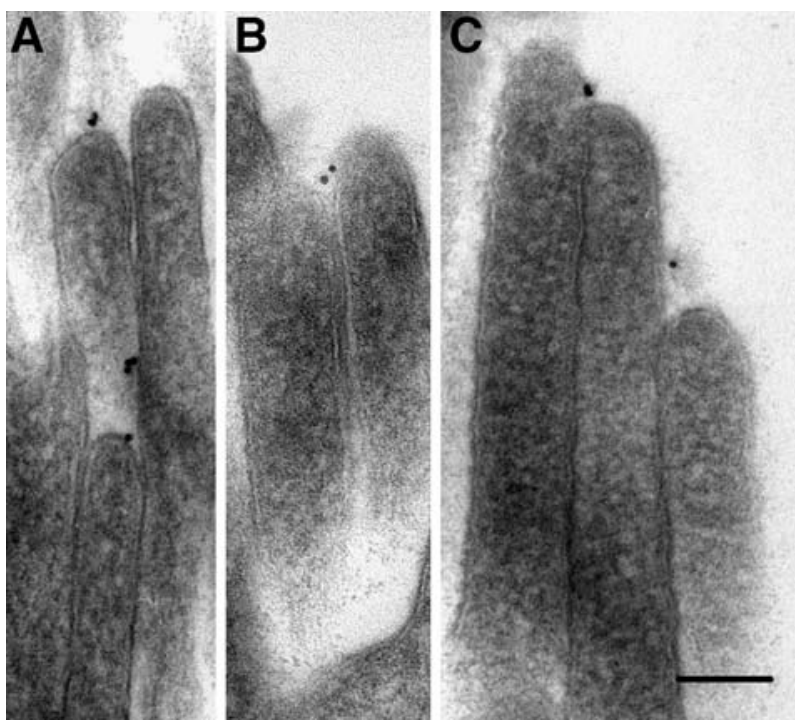

FIG. 9. Immunoelectron microscopy to anti-Arl-5b. Specimens were processed for preembedment immunoelectron microscopy. Healthy control animals were fixed and then bisected. One half of the tissue was exposed to anti-Arl-5b and one half of the tissue was exposed to preimmune serum. Only the tissue exposed to anti-Arl-5b is shown. Small-diameter stereocilia are clustered together. A Gold particles are visible at the tip of a tall stereocilium and at the tip of a short stereocilium. In addition, gold particles occur on the side of a tall stereocilium approximately $100 \mathrm{~nm}$ above the tip of the short stereocilium. B Gold particles occur at the tip of a stereocilium. C Gold particles occur near to the tips of two stereocilia. Scale bar $=110 \mathrm{~nm}$.

stereocilia splay to greatly disorganize the hair bundle. It follows logically that reestablishing linkages into damaged hair bundles may be required to reestablish an orderly array of stereocilia (i.e., a bundle). In immunoelectron microscopy of healthy control animals, colloidal gold particles were observed along the length of stereocilia, including regions in the vicinity of tip links. Of 988 gold particles counted on the surface of anemone tissue, 99 occurred on tissue exposed to preimmune serum. Thus, in specimens exposed to anti-Arl-5b, 90\% of the gold particles likely indicate specific binding sites for anti-Arl-5b. Hence, it appears that Arl-5b is in the correct position to interact with linkages

FIG. 8. Effects of Arl-5b antiserum on hair bundle morphology during the spontaneous recovery to trauma. Anemones were traumatized in calcium-depleted seawater for $1 \mathrm{~h}$, and then were transferred to calcium-containing seawater where they remained for $1 \mathrm{~h}$ before they were fixed and processed for phalloidin cytochemistry. During the recovery, experimental animals were incubated in Arl-5b antiserum diluted in seawater to $1 / 500$ (subpanel A). Control animals were incubated in preimmune serum from the same rabbit diluted to $1 / 500$ (subpanel B). A The recovering hair bundles (hb) apparently consist mostly, or entirely, of large-diameter stereocilia from the sensory neuron (i.e., bundles consist of approximately six stereocilia). The large-diameter stereocilia are markedly splayed. B The recovering hair bundles (hb) consist of a mixture of large-diameter stereocilia and small-diameter stereocilia (i.e., the bundles consist of more than six stereocilia). Scale bar $=15 \mu \mathrm{m}$. critical to hair bundle function. We hypothesize that Arl-5b is involved in maintaining linkages and/or in installing replacement linkages between stereocilia of hair bundle mechanoreceptors.

Finally, given that Arfs can serve as cofactors in pathways for ADP-ribosylation of substrates, we tested whether ADP-ribosyltransferases might be involved in the repair of hair bundles. ADP-ribosyltransferases cleave $\beta$-NAD into nicotinamide and ADP-ribose. The latter is transferred to the substrate protein, typically at arginine residues, to change the activity of the substrate (i.e., to activate or to inhibit the substrate) (Glowacki et al. 2002). Interestingly, exogenously supplied $\beta$-NAD enhanced the activity of fraction $\beta$ so that a full recovery of vibration sensitivity was restored to anemones within 2-3 min rather than 7-8 min of exposure to fraction $\beta$ alone (Watson et al. 1998). In vertebrates, mono-ADP-ribosyltransferases are all ectoenzymes (Glowacki et al. 2002). Their distribution is restricted insofar as they are present in some tissues and absent from others. It is not known whether they occur in cochlea. In muscle, monoADP-ribosyltransferases participate in cellular responses to local tissue injury by adding ADP-ribose to arginine residues of extracellular domains of P2X7 purinoceptors and to integrins. ADP-ribosylation activates the purinoceptors and integrins (Zolkiewska

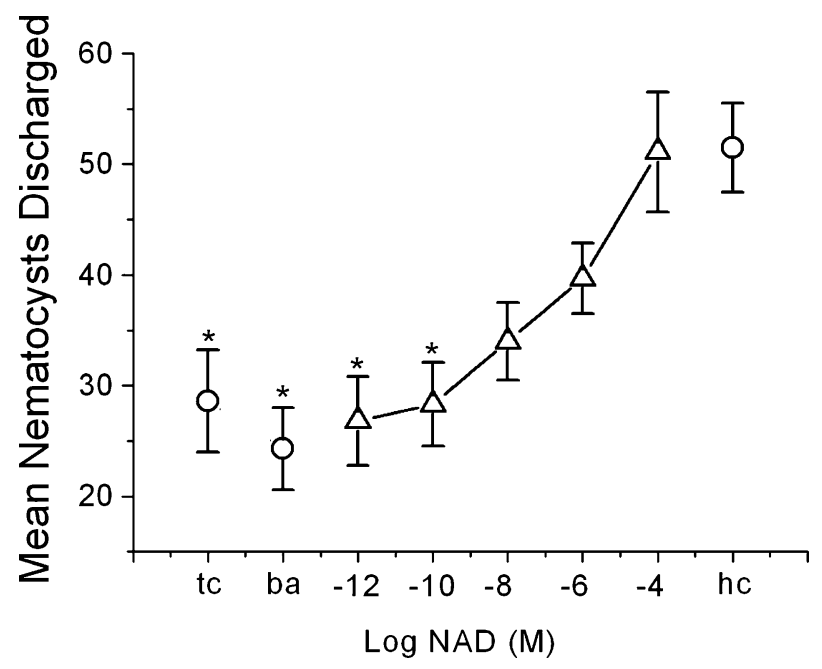

FIG. 10. Effects of exogenous $\beta-N A D$ on vibration-dependent discharge of nematocysts. Specimens were traumatized (or not, healthy controls [hc]) by 1 -h exposure to calcium-depleted seawater. The animals were returned to calcium-containing seawater where they were tested for vibration-dependent discharge of nematocysts at $55 \mathrm{~Hz}$ without further treatment (traumatized controls [tc]) or after 2-min exposure to fraction $\beta$ alone (ba) or in combination with $\beta-N A D$ at the concentration indicated on the $x$-axis. Data points indicate the mean number of nematocysts \pm SEM counted for eight replicate test probes, each touched to a different anemone. Statistical comparisons relative to healthy controls were made using post hoc tests after the ANOVA with significant differences indicated by asterisks. 
$2005)$. The necessary $\beta-\mathrm{NAD}$ is likely exported through connexin-43 hemichannels (Zolkiewska 2005).

There are some striking parallels between injured muscle cells and traumatized hair cells in anemones. First, evidence of ADP-ribosyltransferase involvement in hair cell repair is presented in this study. Secondly, ATP is released into the extracellular space during the repair of hair bundles of sea anemones (Watson et al. 1999). Third, purinoceptors are apparently involved in the repair process (Watson 2000). Finally, connexin-43 is present in hair cells of anemones (Mire et al. 2000). Hence, although direct evidence is not yet available to link the pieces together, a model is emerging in which trauma to hair cells induces an externalization of Arl-5b. The Arl-5b may activate putative ecto-ADP-ribosyltransferases that transfer ADP-ribose to substrates, including P2X purinoceptors. Based on some unpublished observations (GM Watson), it appears that the activated purinoceptors induce a reorganization of the actin cytoskeleton within stereocilia. Cellular stores of $\beta$-NAD needed for the ADP-ribosyltransferases and ATP to bind the purinoceptors may be released into the extracellular space from the cytoplasm through connexin- 43 hemichannels. The Arl-5b also is somehow involved in the replacement or repair of linkages interconnecting stereocilia. Although far from complete, this model constitutes a reasonable starting point for increasing our understanding of the subcellular basis for self-repair of hair cells.

\section{ACKNOWLEDGMENTS}

We appreciate financial support from the NOHR Foundation and NSF IOB0542574 and thoughtful comments on the original manuscript by two anonymous reviewers.

\section{REFERENCES}

Assad JA, Shepherd GM, Corey DP. Tip-link integrity and mechanical transduction in vertebrate hair cells. Neuron. 7:985-994, 1991.

Baird RA, Burton MD, Fashena DS, Naegler RA. Hair cell recovery in mitotically blocked cultures of the bullfrog saccule. Proc. Natl. Acad. Sci. USA. 97:11722-11729, 2000.

Clatrk JA, Pickles JO. The effects of moderate and low levels of acoustic overstimulation on stereocilia and their tip links in the guinea pig. Hear. Res. 99:119-128, 1996.

Darling JA, Reitzel AR, Burton PM, Mazza ME, Ryan JF, Sullivan JC, Finnerty JR. Rising starlet: The starlet sea anemone Nematostella vectensis. BioEssays 27:211-221, 2005.

Duncan RK, Hernandez HN, Saunders JC. Relative stereocilia motion of chick cochlear hair cells during high-frequency water-jet stimulation. Audit. Neurosci. 1:321-329, 1995.

Duncan RK, SAunders JC. Stereocilium injury mediates hair bundle stiffness loss and recovery following intense water-jet stimula- tion. J. Comp. Physiol., A Sens. Neural Behav. Physiol. 186:10951106, 2000.

Gale Je, Marcotti W, Kennedy HJ, Kros CJ, Richardson GP. FM1-43 dye behaves as a permeant blocker of the hair cell mechanotransducer channel. J. Neurosci. 21:7013-7025, 2001.

Glowacki G, Braren R, Firner K, Nissen M, Rede P, Bazan F, Cetrovic-Cvrlje M, Leiter E, Henag F, Koch-Nelte F. The family of toxin-related ecto-ADP ribosyltransferases in humans and the mouse. Protein Sci. 11:1657-1670, 2002.

GOLDBERG J. Structural basis for activation of ARF GTPase: mechanisms of guanine nucleotide exchange and GTP-myristoyl switching. Cell 95:237-248, 1998.

Gong TW, Hegeman AD, Shin JJ, Adler HJ, Raphael Y, Lomax Mi. Identification of genes expressed after noise exposure in the chick basilar papilla. Hear. Res. 96:20-32, 1996.

Goodyear R, Richardson GP. The ankle-link antigen: An epitope sensitive to calcium chelation associated with the hair cell surface and the calycal processes of photoreceptors. J. Neurosci. 19:3761-3772, 1999.

HALl A. Rho GTPases and the actin cytoskeleton. Science 279:509$514,1998$.

Husbands JM, Steinberg SA, Kurian R, Saunders JC. Tip-link integrity on chick tall hair cell stereocilia following intense sound exposure. Hear. Res. 135:135-145, 1999.

Ingham PW, McMahon AP. Hedgehog signaling in animal development: paradigms and principles. Genes Dev. 15:3059-3087, 2001.

Kahn RA, Cherfils J, Elias M, Lovering RC, Munro S, Schurmann A. Nomenclature for the human Arf family of GTP-binding proteins: ARF, ARL, and SAR proteins. J. Cell Biol. 172:645-650, 2006.

Kohtz JD, Lee HY, Gaiano N, Segal J, Ng E, Larson T, Baker DP, Garber EA, Williams KP, Fishell G. N-terminal fatty acylation of sonic hedgehog enhances the induction of rodent forebrain neurons. Development 128:2351-2363, 2001.

Mariscal RN. Nematocysts. In: Muscatine L and Lenhoff HM (eds) Coelenterate Biology, Reviews and New Perspectives. New York, Academic Press, pp. 129-178, 1974.

Meyers JR, MacDonald RB, Duggan A, Lenzi D, Standarct DG, CORWIN JT, COREY DP. Lighting up the senses: FM1-43 loading of sensory cells through nonselective ion channels. J. Neurosci. 23:4054-4065, 2003.

MiNASIAN LL JR, MARISGAL RN. Characteristics and regulation of fission activity in clonal cultures of the cosmopolitan sea anemone, Haliplanella luciae (Verrill). Biol. Bull. 157:478-493, 1979.

Mire P, WATSON GM. Mechanotransduction of hair bundles arising from multicellular complexes in anemones. Hear. Res. 113:224234, 1997.

Mire P, Nasse J, Venable-Thibodeaux S. Gap junctional communication in the vibration-sensitive response of sea anemones. Hear. Res. 144:109-123, 2000.

Oh SH, Adler HJ, Raphael Y, Lomax MI. WDR1 colocalizes with $\mathrm{ADF}$ and actin in the normal and noise-damaged chick cochlea. J. Comp. Neurol. 448:399-409, 2002.

Pickles JO, Osborne MP, Comis SD. Vulnerability of tip links between stereocilia to acoustic trauma in the guinea pig. Hear. Res. 25:173-183, 1987.

Rzadzinska AK, Schneider ME, Davies C, Riordan GP, Kachar B. An actin molecular treadmill and myosins maintain stereocilia functional architecture and self-renewal. J. Cell Biol. 164:887-897, 2004.

Sobkowicz HM, August BK, SLAPNICK SM. Epithelial repair following mechanical injury of the developing organ of Corti in culture: an electron microscopic and autoradiographic study. Exp. Neurol. 115:44-49, 1992.

WATSON GM. How a living hair cell repairs itself: Involvement of purinoceptors in the repair of hair bundle mechanoreceptors of sea anemones. In: Berlin CI (ed) Hair Cell Micromechanics and Hearing. New York, Singular Press, pp. 27-43, 2000. 
Watson GM, Hessinger DA. Cnidocyte mechanoreceptors are tuned to the movements of swimming prey by chemoreceptors. Science 243:1589-1591, 1989.

WATSON GM, Mire P. Reorganization of actin during repair of hair bundle mechanoreceptors. J. Neurocytol. 30:895-906, 2001.

Watson GM, Mire P. Dynamic tuning of hair bundle mechanoreceptors in a sea anemone during predation. Hydrobiologica 530/531:123-128, 2004.

Watson GM, Venable-Thibodeaux S. Immunological evidence that anemone repair proteins include replacement linkages. Hear. Res. 146:35-46, 2000.

Watson GM, Hudson RR. Frequency and amplitude tuning of nematocyst discharge by proline. J. Exp. Zool. 268:177-185, 1994.
Watson GM, Mire P, Hudson RR. Hair bundles of sea anemones as a model system for vertebrate hair bundles. Hear. Res. 107:5366, 1997.

Watson GM, Mire P, Hudson RR. Repair of hair bundles in sea anemones by secreted proteins. Hear. Res. 115:119-128, 1998.

Watson GM, Venable S, Hudson RR, Repass JJ. ATP enhances repair of hair bundles in sea anemones. Hear. Res. 136: 1-12, 1999.

Zhao Y, Yamoah EN, Gillespie PG. Regeneration of broken tip links and restoration of mechanical transduction in hair cells. Proc. Natl. Acad. Sci. USA. 94:15469-15474, 1996.

Zolkiewska A. Ecto-ADP-ribose transferases: cell-surface response to local tissue injury. Physiology. 20:374-381, 2005. 